\title{
BLENDING TYPE APPROXIMATION BY GENERALIZED BERNSTEIN-DURRMEYER TYPE OPERATORS
}

\author{
ARUN KAJLA AND TUNCER ACAR
}

Received 27 January, 2017

\begin{abstract}
In this article we construct a Durrmeyer modification of the operators introduced by Chen et al. in [10] based on a non-negative real parameter. We establish local approximation, global approximation, Voronovskaja type asymptotic theorem. The rate of convergence for differentiable functions whose derivatives are of bounded variation is also obtained.
\end{abstract}

2010 Mathematics Subject Classification: 41A25; 26A15

Keywords: local approximation, global approximation, asymptotic formula, bounded variation

\section{INTRODUCTION}

The approximation theory by linear positive operators investigates how the functions can be best approximated by simpler functions. The most famous basic result for convergence of linear positive operators is due to Weierstrass who introduced an important theorem named Weierstrass' approximation theorem. At last in 1912 Bernstein introduced the most famous algebraic polynomials $B_{n}(f ; x)$ in approximation theory in order to give a constructive proof of Weierstrass' theorem, which are given by

$$
B_{n}(f ; x)=\sum_{k=0}^{n} p_{n, k}(x) f\left(\frac{k}{n}\right), x \in[0,1],
$$

where $p_{n, k}(x)=\left(\begin{array}{l}n \\ k\end{array}\right) x^{k}(1-x)^{n-k}$ and he proved that if $f \in C[0,1]$ then $B_{n}(f ; x)$ converges uniformly to $f(x)$ in $[0,1]$.

The Bernstein operators have been used in many branches of mathematics and computer science. Due to their useful structure, Bernstein polynomials and their modifications have been intensively studied. Among other papers, we refer the readers to $[3,7,9,13,23,25]$. 
For $f \in C[0,1]$, Chen et al. in [10] introduced a generalization of the Bernstein operators based on a non-negative parameter $\alpha(0 \leq \alpha \leq 1)$ as follows:

$$
T_{n}^{(\alpha)}(f ; x)=\sum_{k=0}^{n} p_{n, k}^{(\alpha)}(x) f\left(\frac{k}{n}\right), \quad x \in[0,1]
$$

where

$$
\begin{aligned}
& p_{n, k}^{(\alpha)}(x)= \\
& {\left[\left(\begin{array}{c}
n-2 \\
k
\end{array}\right)(1-\alpha) x+\left(\begin{array}{l}
n-2 \\
k-2
\end{array}\right)(1-\alpha)(1-x)+\left(\begin{array}{l}
n \\
k
\end{array}\right) \alpha x(1-x)\right] x^{k-1}(1-x)^{n-k-1}}
\end{aligned}
$$

and $n \geq 2$. They proved the rate of convergence, Voronovskaja type asymptotic formula and shape preserving properties for these operators. For the special case, $\alpha=1$, these operators reduce the well-known Bernstein operators.

The Durrmeyer type modification of the operators is a method to approximate the Lebesgue integrable functions. For this aim, many researchers have studied in this direction. Gupta and Rassias [18] introduced the Lupas-Durrmeyer type operators based on Polya distribution and established asymptotic approximation, local and global results. Goyal et al. [14] considered a one parameter family of BaskakovSzász type operators and studied quantitative convergence theorems for these operators. Gupta et al. [16] introduced hybrid operators involving inverse PolyaEggenberger distribution and studied degree of approximation of these operators which include global approximation and uniform convergence. Very recently, Acu and Gupta [15] defined a summation-integral type operators depending on two parameters and discussed some approximation results e.g. local approximation, Voronovskaja type asymtotic theorem and weighted approximation of these operators. In the literature survey, several authors have studied the approximation behavior of mixed hybrid operators (cf. [1,2,4-6,8,17, 19-21]).

Inspired by their work, for $f \in C[0,1]$ we define the following Durrmeyer type modification of the operators (1.1) as:

$$
D_{n}^{(\alpha)}(f ; x)=(n+1) \sum_{k=0}^{n} p_{n, k}^{(\alpha)}(x) \int_{0}^{1} p_{n, k}(t) f(t) d t, \quad x \in[0,1] .
$$

The purpose of this paper is to study the Voronovskaja type theorem, local approximation, pointwise estimates and global approximation results for these operators (1.3). The rate of convergence for differentiable functions whose derivatives are of bounded variation is also obtained.

\section{BASIC RESULTS}

In what follows let $\|\cdot\|$ denote the uniform norm on $C[0,1]$. 
Let $e_{i}=t^{i}, i \in \mathbb{N} \cup\{0\}$. By simple computation, we get

$$
\int_{0}^{1} p_{n, k}(t) t^{i} d t=\frac{n !(k+i) !}{k !(n+i+1) !} .
$$

In order to prove the main results, we will show some lemmas in this section. We need the following auxiliary results.

Lemma 1 ([10]). For the operators $T_{n}^{(\alpha)}(f ; x)$, we have

(i) $T_{n}^{(\alpha)}\left(e_{0} ; x\right)=1$;

(ii) $T_{n}^{(\alpha)}\left(e_{1} ; x\right)=x$;

(iii) $T_{n}^{(\alpha)}\left(e_{2} ; x\right)=x^{2}+\frac{(n+2(1-\alpha))}{n^{2}} x(1-x)$;

(iv) $T_{n}^{(\alpha)}\left(e_{3} ; x\right)=x^{3}+\frac{3(n+2(1-\alpha))}{n^{2}} x^{2}(1-x)$

$+\frac{(n+6(1-\alpha))}{n^{3}} x(1-x)(1-2 x)$

(v) $T_{n}^{(\alpha)}\left(e_{4} ; x\right)=x^{4}+\frac{6(n+2(1-\alpha))}{n^{2}} x^{3}(1-x)$

$+\frac{4(n+6(1-\alpha))}{n^{3}} x^{2}(1-x)(1-2 x)$

$+\frac{((3 n(n-2)+12(n-6)(1-\alpha)) x(1-x)+(n+14(1-\alpha)))}{n^{4}} x(1-x)$.

Lemma 2. For the operators $D_{n}^{(\alpha)}(f ; x)$, we have

(i) $D_{n}^{(\alpha)}\left(e_{0} ; x\right)=1$;

(ii) $D_{n}^{(\alpha)}\left(e_{1} ; x\right)=x+\frac{1-2 x}{(n+2)}$;

(iii) $D_{n}^{(\alpha)}\left(e_{2} ; x\right)=x^{2}+\frac{2 x^{2}(\alpha-3 n-4)}{(n+2)(n+3)}+\frac{2 x(2 n-\alpha+1)}{(n+2)(n+3)}+\frac{2}{(n+2)(n+3)}$;

(iv) $D_{n}^{(\alpha)}\left(e_{3} ; x\right)=x^{3}+\frac{6 x^{3}(-n(5+2 n-\alpha)-2(1+\alpha))}{(n+2)(n+3)(n+4)}$

$+\frac{3 x^{2}(n(3 n-2 \alpha-1)+10(\alpha-1))}{(n+2)(n+3)(n+4)}+\frac{18 x(n-\alpha+1)}{(n+2)(n+3)(n+4)}$

$+\frac{6}{(n+2)(n+3)(n+4)}$

(v) $D_{n}^{(\alpha)}\left(e_{4} ; x\right)=x^{4}+\frac{x^{4}(-4(n+3)(16+n(3+5 n))+12 \alpha(n-3)(n-2))}{(n+2)(n+3)(n+4)(n+5)}$

$+\frac{4 x^{3}(n-2)(n(4 n-3 \alpha-1)+33(\alpha-1))}{(n+2)(n+3)(n+4)(n+5)}$ 


$$
\begin{aligned}
& +\frac{24 x^{2}\left(n+3 n^{2}+14(\alpha-1)-4 n \alpha\right)}{(n+2)(n+3)(n+4)(n+5)}+\frac{48 x(2 n-3 \alpha+3)}{(n+2)(n+3)(n+4)(n+5)} \\
& +\frac{24}{(n+2)(n+3)(n+4)(n+5)} .
\end{aligned}
$$

Proof. This lemma follows easily applying Lemma 1 and relation (2.1). Hence the details are omitted.

Lemma 3. From Lemma 2, we get

(i) $D_{n}^{(\alpha)}(t-x ; x)=\frac{1-2 x}{n+2}$;

(ii) $D_{n}^{(\alpha)}\left((t-x)^{2} ; x\right)=\frac{2 x(1-x)(n-\alpha-2)}{(n+2)(n+3)}+\frac{2}{(n+2)(n+3)}$;

(iii) $D_{n}^{(\alpha)}\left((t-x)^{4} ; x\right)=\frac{12 x^{3}(x-2)(n(n-2 \alpha-19)+46 \alpha-36)}{(n+2)(n+3)(n+4)(n+5)}$

$$
\begin{aligned}
& +\frac{12 x^{2}(n(n-2 \alpha-25)+58 \alpha-38)}{(n+2)(n+3)(n+4)(n+5)}+\frac{24 x(3 n-6 \alpha+1)}{(n+2)(n+3)(n+4)(n+5)} \\
& +\frac{24}{(n+2)(n+3)(n+4)(n+5)} .
\end{aligned}
$$

Lemma 4. For $f \in C[0,1]$, we have $\left\|D_{n}^{(\alpha)}(f ; \cdot)\right\| \leq\|f\|$.

Proof. From definition (1.3) and Lemma 2, we have

$$
\begin{aligned}
\left\|D_{n}^{(\alpha)}(f ; \cdot)\right\| & \leq(n+1) \sum_{k=0}^{n} p_{n, k}^{(\alpha)}(x) \int_{0}^{1} p_{n, k}(t)|f(t)| d t \\
& \leq\|f\| D_{n}^{(\alpha)}\left(e_{0} ; x\right)=\|f\| .
\end{aligned}
$$

Lemma 5. For $n \in \mathbb{N}$, we have

$$
D_{n}^{(\alpha)}\left((t-x)^{2} ; x\right) \leq \frac{2 \gamma_{n}^{2}(x)}{(n+2)},
$$

where $\gamma_{n}^{2}(x)=\varphi^{2}(x)+\frac{1}{(n+2)}$ and $\varphi^{2}(x)=x(1-x)$.

Proof. This result is obtained by straightforward computation, but the details are omitted.

Remark 1. Let $\Theta_{n}^{\alpha, m}:=D_{n}^{(\alpha)}\left((t-x)^{m} ; x\right), m=1,2,4$ be the central moments of $D_{n}^{(\alpha)}$, we get

$$
\begin{aligned}
& \lim _{n \rightarrow \infty} n \Theta_{n}^{\alpha, 1}(x)=1-2 x, \\
& \lim _{n \rightarrow \infty} n \Theta_{n}^{\alpha, 2}(x)=2 x(1-x),
\end{aligned}
$$




$$
\lim _{n \rightarrow \infty} n^{2} \Theta_{n}^{\alpha, 4}(x)=12 x^{2}(1-x)^{2} .
$$

\section{BASIC CONVERGENCE THEOREM}

Theorem 1. Suppose that $f \in C[0,1]$ and $\alpha \in[0,1]$. Then $\lim _{n \rightarrow \infty} D_{n}^{(\alpha)}(f ; x)=$ $f(x)$, uniformly in $[0,1]$.

Proof. Applying Lemma $2, D_{n}^{(\alpha)}\left(e_{0} ; x\right)=1, D_{n}^{(\alpha)}\left(e_{1} ; x\right) \rightarrow x, D_{n}^{(\alpha)}\left(e_{2} ; x\right) \rightarrow x^{2}$ as $n \rightarrow \infty$, uniformly in [0,1]. By the well-known Bohman-Korovkin theorem, it follows that $D_{n}^{(\alpha)}(f ; x) \rightarrow f(x)$ as $n \rightarrow \infty$, uniformly in [0,1].

\section{LOCAL APPROXIMATION}

The $K$-functional is given by :

$$
K_{2}(f, \delta)=\inf \left\{\|f-g\|+\delta\left\|g^{\prime \prime}\right\|: g \in W^{2}\right\}(\delta>0),
$$

where $W^{2}=\left\{g: g^{\prime \prime} \in C[0,1]\right\}$ and $\|$.$\| is the uniform norm on C[0,1]$. By [11] there exists a positive constant $M>0$ such that

$$
K_{2}(f, \delta) \leq M \omega_{2}(f, \sqrt{\delta}),
$$

where the second order modulus of continuity for $f \in C[0,1]$ is defined as

$$
\omega_{2}(f, \sqrt{\delta})=\sup _{0<h \leq \sqrt{\delta} x, x+2 h \in[0,1]}|f(x+2 h)-2 f(x+h)+f(x)| .
$$

We define the usual modulus of continuity for $f \in C[0,1]$ as

$$
\omega(f, \delta)=\sup _{0<h \leq \delta} \sup _{x, x+h \in[0,1]}|f(x+h)-f(x)| .
$$

Theorem 2. For the operators $D_{n}^{(\alpha)}$, there exists a constant $M>0$ such that

$$
\left|D_{n}^{(\alpha)}(f ; x)-f(x)\right| \leq M \omega_{2}\left(f,(n+2)^{-1 / 2} \gamma_{n}(x)\right)+\omega\left(f,\left|\frac{1-2 x}{n+2}\right|\right),
$$

where $f \in C[0,1], \alpha \in[0,1], \gamma_{n}^{2}(x)=\varphi^{2}(x)+\frac{1}{(n+2)}$ and $x \in[0,1]$.

Proof. We define the auxiliary operators as follows:

$$
\bar{D}_{n}^{(\alpha)}(f ; x)=D_{n}^{(\alpha)}(f ; x)+f(x)-f\left(\frac{n x+1}{n+2}\right) .
$$

Then, we can easily check that

$$
\bar{D}_{n}^{(\alpha)}(1 ; x)=1 \text { and } \bar{D}_{n}^{(\alpha)}(t ; x)=x .
$$

Let $g \in W^{2}$ and $t \in[0,1]$. By Taylor's expansion we have

$$
g(t)=g(x)+(t-x) g^{\prime}(x)+\int_{x}^{t}(t-u) g^{\prime \prime}(u) d u .
$$


Applying the operator $\bar{D}_{n}^{(\alpha)}$ on both sides of the above relation, we may write

$$
\begin{aligned}
\bar{D}_{n}^{(\alpha)}(g ; x)= & g(x)+\bar{D}_{n}^{(\alpha)}\left(\int_{x}^{t}(t-u) g^{\prime \prime}(u) d u\right) \\
= & g(x)+D_{n}^{(\alpha)}\left(\int_{x}^{t}(t-u) g^{\prime \prime}(u) d u, x\right) \\
& -\int_{x}^{\frac{n x+1}{(n+2)}}\left(\frac{n x+1}{n+2}-u\right) g^{\prime \prime}(u) d u .
\end{aligned}
$$

Hence

$$
\begin{aligned}
& \left|\bar{D}_{n}^{(\alpha)}(g ; x)-g(x)\right| \leq D_{n}^{(\alpha)}\left(\left|\int_{x}^{t}\right| t-u|| g^{\prime \prime}(u)|d u|, x\right) \\
& +\left|\int_{x}^{\frac{n x+1}{(n+2)}}\right| \frac{n x+1}{n+2}-u|| g^{\prime \prime}(u)|d u| \\
& \leq\left\{D_{n}^{(\alpha)}\left((t-x)^{2} ; x\right)+\left(\frac{n x+1}{n+2}-x\right)^{2}\right\}\left\|g^{\prime \prime}\right\| \\
& =\left\{D_{n}^{(\alpha)}\left((t-x)^{2} ; x\right)+\left(\frac{1-2 x}{n+2}\right)^{2}\right\}\left\|g^{\prime \prime}\right\| \text {. }
\end{aligned}
$$

From Lemma 5, we have

$$
\begin{aligned}
D_{n}^{(\alpha)}\left((t-x)^{2} ; x\right)+\left(\frac{1-2 x}{n+2}\right)^{2} & \leq \frac{2}{(n+2)} \gamma_{n}^{2}(x)+\left(\frac{1-2 x}{n+2}\right)^{2} \\
& \leq \frac{2}{(n+2)} \gamma_{n}^{2}(x)+\frac{1}{(n+2)^{2}} \\
& \leq \frac{3}{(n+2)} \gamma_{n}^{2}(x) .
\end{aligned}
$$

Thus, by (4.2) we have

$$
\left|\bar{D}_{n}^{(\alpha)}(g ; x)-g(x)\right| \leq \frac{3}{(n+2)} \gamma_{n}^{2}(x)|| g^{\prime \prime} \|,
$$

where $x \in[0,1]$. Furthermore, by Lemma 4, we have

$$
\left|\bar{D}_{n}^{(\alpha)}(f ; x)\right| \leq 3\|f\|,
$$

for all $f \in C[0,1]$ and $x \in[0,1]$.

Now, for $f \in C[0,1]$ and $g \in W^{2}$, using (4.4) and (4.5) we obtain that

$$
\left|D_{n}^{(\alpha)}(f ; x)-f(x)\right| \leq\left|\bar{D}_{n}^{(\alpha)}(f ; x)-f(x)+f\left(\frac{n x+1}{n+2}\right)-f(x)\right|
$$




$$
\begin{aligned}
\leq & \left|\bar{D}_{n}^{(\alpha)}(f-g ; x)\right|+\left|\bar{D}_{n}^{(\alpha)}(g ; x)-g(x)\right|+|g(x)-f(x)| \\
& +\left|f\left(\frac{n x+1}{n+2}\right)-f(x)\right| \\
\leq & 4|| f-g \|+\frac{3}{(n+2)} \gamma_{n}^{2}(x)|| g^{\prime \prime}||+\omega\left(f,\left|\frac{1-2 x}{n+2}\right|\right) .
\end{aligned}
$$

Taking the infimum on the right hand side over all $g \in W^{2}$, we get

$$
\left|D_{n}^{(\alpha)}(f ; x)-f(x)\right| \leq 4 K_{2}\left(f, \frac{1}{(n+2)} \gamma_{n}^{2}(x)\right)+\omega\left(f,\left|\frac{1-2 x}{n+2}\right|\right) .
$$

Now considering the relation (4.1), we obtain

$$
\left|D_{n}^{(\alpha)}(f ; x)-f(x)\right| \leq M \omega_{2}\left(f,(n+2)^{-1 / 2} \gamma_{n}(x)\right)+\omega\left(f,\left|\frac{1-2 x}{n+2}\right|\right),
$$

which completes the proof.

Let $a_{1} \geq 0, a_{2}>0$ and let us now consider the Lipschitz-type space [24]:

$$
\operatorname{Lip}_{M}^{*}(\rho):=\left\{f \in C[0,1]:|f(t)-f(x)| \leq M \frac{|t-x|^{\rho}}{\left(t+a_{1} x^{2}+a_{2} x\right)^{\frac{\rho}{2}}} ; x, t \in(0,1)\right\},
$$

where $\rho \in(0,1]$.

Theorem 3. Let $f \in \operatorname{Lip}_{M}^{*}(\rho)$. Then, for all $x \in(0,1]$, we have

$$
\left|D_{n}^{(\alpha)}(f ; x)-f(x)\right| \leq M\left(\frac{\Theta_{n}^{\alpha, 2}(x)}{a_{1} x^{2}+a_{2} x}\right)^{\frac{\rho}{2}},
$$

where $\Theta_{n}^{\alpha, 2}(x)=D_{n}^{(\alpha)}\left((t-x)^{2} ; x\right)$.

Proof. First, we show the result for the case $\rho=1$. We may write

$$
\begin{aligned}
\left|D_{n}^{(\alpha)}(f ; x)-f(x)\right| & \leq(n+1) \sum_{k=0}^{n} p_{n, k}^{(\alpha)}(x) \int_{0}^{1}|f(t)-f(x)| d t \\
& \leq M(n+1) \sum_{k=0}^{n} p_{n, k}^{(\alpha)}(x) \int_{0}^{1} p_{n, k}(t) \frac{|t-x|}{\sqrt{\left(t+a_{1} x^{2}+a_{2} x\right)}} d t .
\end{aligned}
$$

Using the fact that $\frac{1}{\sqrt{\left(t+a_{1} x^{2}+a_{2} x\right)}}<\frac{1}{\sqrt{\left(a_{1} x^{2}+a_{2} x\right)}}$ and the Cauchy-Schwarz inequality, we have

$$
\left|D_{n}^{(\alpha)}(f ; x)-f(x)\right| \leq \frac{M(n+1)}{\sqrt{\left(a_{1} x^{2}+a_{2} x\right)}} \sum_{k=0}^{n} p_{n, k}^{(\alpha)}(x) \int_{0}^{1} p_{n, k}(t)|t-x| d t
$$




$$
=\frac{M}{\sqrt{\left(a_{1} x^{2}+a_{2} x\right)}} D_{n}^{(\alpha)}(|t-x| ; x) \leq M\left(\sqrt{\frac{\Theta_{n}^{\alpha, 2}(x)}{a_{1} x^{2}+a_{2} x}}\right),
$$

hence the result is obtained for $\rho=1$. Now, we prove the theorem for the case $0<\rho<1$. By the Hölder's inequality with $p=\frac{1}{\rho}$ and $q=\frac{1}{1-\rho}$, we get

$$
\begin{aligned}
\left|D_{n}^{(\alpha)}(f ; x)-f(x)\right| & \leq(n+1) \sum_{k=0}^{n} p_{n, k}^{(\alpha)}(x) \int_{0}^{1} p_{n, k}(t)|f(t)-f(x)| d t \\
& \leq\left\{\sum_{k=0}^{n} p_{n, k}^{(\alpha)}(x)\left((n+1) \int_{0}^{1} p_{n, k}(t)|f(t)-f(x)| d t\right)^{\frac{1}{\rho}}\right\}^{\rho} \\
& \leq\left\{(n+1) \sum_{k=0}^{n} p_{n, k}^{(\alpha)}(x) \int_{0}^{1} p_{n, k}(t)|f(t)-f(x)|^{\frac{1}{\rho}} d t\right\}^{\rho} \\
& \leq M\left\{(n+1) \sum_{k=0}^{n} p_{n, k}^{(\alpha)}(x) \int_{0}^{1} p_{n, k}(t) \frac{|t-x|}{\sqrt{\left(t+a_{1} x^{2}+a_{2} x\right)}} d t\right\}^{\rho} \\
& \leq \frac{M}{\left(a_{1} x^{2}+a_{2} x\right)^{\frac{\rho}{2}}}\left\{(n+1) \sum_{k=0}^{n} p_{n, k}^{(\alpha)}(x) \int_{0}^{1} p_{n, k}(t)|t-x| d t\right\}^{\rho} \\
& \leq \frac{M}{\left(a_{1} x^{2}+a_{2} x\right)^{\frac{\rho}{2}}}\left(D_{n}^{(\alpha)}(|t-x| ; x)\right)^{\rho} \leq M\left(\frac{\Theta_{n}^{\alpha, 2}(x)}{a_{1} x^{2}+a_{2} x}\right)^{\frac{\rho}{2}} .
\end{aligned}
$$

Thus, the proof is completed.

Next, we study the local direct estimate of the operators defined in (1.3) applying the Lipschitz-type maximal function of order $\rho$ given by Lenze [22] as

$$
\widetilde{\omega}_{\rho}(f, x)=\sup _{t \neq x, t \in[0,1]} \frac{|f(t)-f(x)|}{|t-x|^{\rho}}, x \in[0,1] \text { and } \rho \in(0,1] .
$$

Theorem 4. Let $f \in C[0,1]$ and $0<\rho \leq 1$. Then, for all $x \in[0,1]$, we have

$$
\left|D_{n}^{(\alpha)}(f ; x)-f(x)\right| \leq \widetilde{\omega}_{\rho}(f, x)\left\{\frac{2}{(n+2)} \gamma_{n}^{2}(x)\right\}^{\frac{\rho}{2}}
$$

Proof. In view of (4.6), we have

$$
|f(t)-f(x)| \leq \widetilde{\omega}_{\rho}(f, x)|t-x|^{\rho}
$$

and

$$
\left|D_{n}^{(\alpha)}(f ; x)-f(x)\right| \leq D_{n}^{(\alpha)}(|f(t)-f(x)| ; x) \leq \widetilde{\omega}_{\rho}(f, x) D_{n}^{(\alpha)}\left(|t-x|^{\rho} ; x\right) .
$$


Now, applying the Hölder's inequality with $p=\frac{2}{\rho}$ and $\frac{1}{q}=1-\frac{1}{p}$, we have

$$
\begin{aligned}
\left|D_{n}^{(\alpha)}(f ; x)-f(x)\right| & \leq \widetilde{\omega}_{\rho}(f, x) D_{n}^{(\alpha)}\left((t-x)^{2} ; x\right)^{\frac{\rho}{2}} \\
& \leq \widetilde{\omega}_{\rho}(f, x)\left\{\frac{2}{(n+2)} \gamma_{n}^{2}(x)\right\}^{\frac{\rho}{2}} .
\end{aligned}
$$

\section{Global APPROXimation}

Let $f \in C[0,1]$ and $\varphi(x)=\sqrt{x(1-x)}, x \in[0,1]$. The second order Ditzian-Totik modulus of smoothness and corresponding $K$-functional are given by, respectively,

$$
\begin{gathered}
\omega_{2}^{\varphi}(f, \sqrt{\delta})=\sup _{0<h \leq \sqrt{\delta} x \pm h \varphi(x) \in[0,1]} \sup _{0}|f(x+h \varphi(x))-2 f(x)+f(x-h \varphi(x))|, \\
\tilde{K}_{2, \varphi(x)}(f, \delta)=\inf \left\{\|f-g\|+\delta\left\|\varphi^{2} g^{\prime \prime}\right\|+\delta^{2}\left\|g^{\prime \prime}\right\|: g \in W^{2}(\varphi)\right\},(\delta>0),
\end{gathered}
$$

where $W^{2}(\varphi)=\left\{g \in C[0,1]: g^{\prime} \in A C_{l o c}[0,1], \varphi^{2} g^{\prime \prime} \in C[0,1]\right\}$ and $g^{\prime} \in A C_{l o c}[0,1]$ means that $g$ is differentiable and $g^{\prime}$ is absolutely continuous on every closed interval $[a, b] \subset(0,1)$. It is known ([12], Theorem 1.3.1) that there exists a positive constant $M>0$, such that

$$
\tilde{K}_{2, \varphi(x)}(f, \delta) \leq M \omega_{2}^{\varphi}(f, \sqrt{\delta}) .
$$

Also, the Ditzian-Totik modulus of first order is given by

$$
\overrightarrow{\omega_{\psi}}(f, \delta)=\sup _{0<h \leq \delta} \sup _{x \pm \frac{h}{2} \psi(x) \in[0,1]}\left|f\left(x+\frac{h}{2} \psi(x)\right)-f\left(x-\frac{h}{2} \psi(x)\right)\right|,
$$

where $\psi:[0,1] \rightarrow \mathbb{R}$ is an admissible step-weight function.

Now we state our next main result.

Theorem 5. Let $f \in C[0,1]$ and $0 \leq \alpha \leq 1$. Then, for $x \in[0,1]$,

$$
\left\|D_{n}^{(\alpha)} f-f\right\| \leq M \omega_{2}^{\varphi}\left(f,(n+2)^{-1 / 2}\right)+\overrightarrow{\omega_{\psi}}\left(f,(n+2)^{-1}\right)+\omega\left(f ;(n+2)^{-1}\right),
$$

where $\varphi^{2}(x)=x(1-x)$ and $\psi(x)= \begin{cases}1-2 x & x \in[0,1 / 2] \\ 2 x-1 & x \in[1 / 2,1]\end{cases}$

Proof. We consider the auxiliary operators as follows:

$$
\bar{D}_{n}^{(\alpha)}(f ; x)=D_{n}^{(\alpha)}(f ; x)+f(x)-f\left(\frac{n x+1}{n+2}\right) .
$$


Let $g \in W^{2}(\varphi)$ then by using Taylor's expansion of $g$, on proceeding as in the proof of Theorem 2, we obtain that

$$
\begin{aligned}
\left|\bar{D}_{n}^{(\alpha)}(g ; x)-g(x)\right| \leq & D_{n}^{(\alpha)}\left(\left|\int_{x}^{t}\right| t-u|| g^{\prime \prime}(u)|d u|, x\right) \\
& +\int_{x}^{\frac{n x+1}{(n+2)}}\left|\frac{n x+1}{n+2}-u\right|\left|g^{\prime \prime}(u)\right| d u .
\end{aligned}
$$

Setting $u=\beta x+(1-\beta) t, \beta \in[0,1]$, and also applying the concavity of $\gamma_{n}^{2}$, we have

$$
\frac{|t-u|}{\gamma_{n}^{2}(u)}=\frac{\beta|t-x|}{\gamma_{n}^{2}(\beta x+(1-\beta) t)} \leq \frac{\beta|t-x|}{\gamma_{n}^{2}(x) \beta+\gamma_{n}^{2}(t)(1-\beta)} \leq \frac{|t-x|}{\gamma_{n}^{2}(x)} .
$$

Thus, inequality (5.2), in view of (5.3) leads us to

$$
\begin{aligned}
\left|\bar{D}_{n}^{(\alpha)}(g ; x)-g(x)\right| \leq & D_{n}^{(\alpha)}\left(\left|\int_{x}^{t} \frac{|t-u|}{\gamma_{n}^{2}(u)} d u\right|, x\right)\left\|\gamma_{n}^{2} g^{\prime \prime}\right\| \\
& +\left(\int_{x}^{\frac{n x+1}{(n+2)}} \frac{\left|\frac{n x+1}{n+2}-u\right|}{\gamma_{n}^{2}(u)} d u\right)\left\|\gamma_{n}^{2} g^{\prime \prime}\right\| . \\
& \leq \frac{1}{\gamma_{n}^{2}(x)}\left\|\gamma_{n}^{2} g^{\prime \prime}\right\|\left[D_{n}^{(\alpha)}\left((t-x)^{2} ; x\right)+\left(\frac{1-2 x}{n+2}\right)^{2}\right] .
\end{aligned}
$$

Now, using inequality (4.3), we get

$$
\begin{aligned}
\left|\bar{D}_{n}^{(\alpha)}(g ; x)-g(x)\right| & \leq \frac{3}{(n+2)}\left\|\gamma_{n}^{2} g^{\prime \prime}\right\| \\
& \leq \frac{3}{(n+2)}\left(\left\|\varphi^{2} g^{\prime \prime}\right\|+\frac{1}{(n+2)}\left\|g^{\prime \prime}\right\|\right) .
\end{aligned}
$$

Applying (4.5) and (5.4), we have for $f \in C[0,1]$,

$$
\begin{aligned}
\left|D_{n}^{(\alpha)}(f ; x)-f(x)\right| \leq & \left|\bar{D}_{n}^{(\alpha)}(f-g, x)\right|+\left|\bar{D}_{n}^{(\alpha)}(g ; x)-g(x)\right|+|g(x)-f(x)| \\
& +\left|f\left(\frac{n x+1}{n+2}\right)-f(x)\right| \\
\leq & 4|| f-g\left\|+\frac{3}{(n+2)}\right\| \varphi^{2} g^{\prime \prime}||+\frac{3}{(n+2)^{2}}\left\|g^{\prime \prime}\right\| \\
& +\left|f\left(\frac{n x+1}{n+2}\right)-f(x)\right|
\end{aligned}
$$

Taking the infimum on the right hand side over all $g \in W^{2}(\varphi)$, we get

$$
\left|D_{n}^{(\alpha)}(f ; x)-f(x)\right| \leq 4 \tilde{K}_{2, \varphi}\left(f, \frac{1}{n+2}\right)+\left|f\left(\frac{n x+1}{n+2}\right)-f(x)\right| .
$$


Also,

$$
\begin{aligned}
\left|f\left(\frac{n x+1}{n+2}\right)-f(x)\right|= & \left|f\left(x+\frac{1-2 x}{n+2}\right)-f(x)\right| \\
\leq & \left|f\left(x+\frac{(1-2 x)}{n+2}\right)-f\left(x-\frac{(1-2 x)}{n+2}\right)\right| \\
& +\left|f\left(x-\frac{(1-2 x)}{n+2}\right)-f(x)\right| \\
\leq & \overrightarrow{\omega_{\psi}}\left(f,(n+2)^{-1}\right)+\omega\left(f ;(n+2)^{-1}\right) .
\end{aligned}
$$

Hence, combining (5.1), (5.5) and (5.6), the desired relation is immediate.

\section{Pointwise COnVERGence of $D_{n}^{(\alpha)}$}

Now we present a Voronovskaja type asymptotic formula for the operators $D_{n}^{(\alpha)}$.

Theorem 6. Let $f \in C[0,1]$ and $\alpha \in[0,1]$. If $f^{\prime}, f^{\prime \prime}$ exists at a point $x \in[0,1]$ then

$$
\lim _{n \rightarrow \infty} n\left(D_{n}^{(\alpha)}(f ; x)-f(x)\right)=(1-2 x) f^{\prime}(x)+x(1-x) f^{\prime \prime}(x),
$$

Further, if $f^{\prime \prime} \in C[0,1]$ then (6.1) holds uniformly in $[0,1]$.

Proof. By Taylor's formula, we can write

$$
f(t)=f(x)+(t-x) f^{\prime}(x)+\frac{1}{2}(t-x)^{2} f^{\prime \prime}(x)+\zeta(t, x)(t-x)^{2},
$$

where $\zeta(t, x) \rightarrow 0$ as $t \rightarrow x$ and is a continuous function on [0,1]. Operating $D_{n}^{(\alpha)}$ to (6.2) and Remark 1, we get

$$
\begin{aligned}
D_{n}^{(\alpha)}(f ; x)-f(x)= & f^{\prime}(x) D_{n}^{(\alpha)}((t-x) ; x)+\frac{1}{2} f^{\prime \prime}(x) D_{n}^{(\alpha)}\left((t-x)^{2} ; x\right) \\
& +D_{n}^{(\alpha)}\left(\zeta(t, x)(t-x)^{2} ; x\right), \\
\lim _{n \rightarrow \infty} n\left(D_{n}^{(\alpha)}(f ; x)-f(x)\right)= & (1-2 x) f^{\prime}(x)+x(1-x) f^{\prime \prime}(x) \\
& +\lim _{n \rightarrow \infty} n D_{n}^{(\alpha)}\left(\zeta(t, x)(t-x)^{2} ; x\right) .
\end{aligned}
$$

Since $\zeta(t, x) \rightarrow 0$ as $t \rightarrow x$, for a given $\epsilon>0$, there exists a $\delta>0$ such that $|\zeta(t, x)|<$ $\epsilon$ whenever $|t-x|<\delta$. For $|t-x| \geq \delta$, we have $|\zeta(t, x)| \leq M \frac{(t-x)^{2}}{\delta^{2}}$, for some $M>$ 0 . Let $\chi_{\delta}(t)$ denote the characteristic function of the interval $(x-\delta, x+\delta)$. From Lemma 3 , we get

$$
\begin{aligned}
\left|D_{n}^{(\alpha)}\left(\zeta(t, x)(t-x)^{2} ; x\right)\right| \leq & D_{n}^{(\alpha)}\left(|\zeta(t, x)|(t-x)^{2} \chi_{\delta}(t) ; x\right) \\
& +D_{n}^{(\alpha)}\left(|\zeta(t, x)|(t-x)^{2}\left(1-\chi_{\delta}(t)\right) ; x\right)
\end{aligned}
$$




$$
\begin{aligned}
& <\epsilon D_{n}^{(\alpha)}\left((t-x)^{2} ; x\right)+\frac{M}{\delta^{2}} D_{n}^{(\alpha)}\left((t-x)^{4} ; x\right) \\
& =\epsilon O\left(\frac{1}{n}\right)+O\left(\frac{1}{n^{2}}\right)
\end{aligned}
$$

which implies that $\lim _{n \rightarrow \infty} n D_{n}^{*(1 / n)}\left(\zeta(t, x)(t-x)^{2} ; x\right)=0$, due to the arbitrariness of $\epsilon>0$. This proves the first assertion of the theorem.

To prove the uniformity assertion, due to the uniform continuity of $f$ in $[0,1]$, the $\delta$ in the above proof can be chosen independent of $x$ and all the other estimates hold uniformly in $x \in[0,1]$.

\section{RATE OF CONVERGENCE}

$D B V[0,1]$ denotes the class of all absolutely continuous functions $f$ defined on $[0,1]$, having on $[0,1]$ a derivative $f^{\prime}$ equivalent to a function of bounded variation on $[0,1]$. We notice that the functions $f \in D B V[0,1]$ possess a representation

$$
f(x)=\int_{0}^{x} g(t) d t+f(0)
$$

where $g \in B V[0,1]$, i.e., $g$ is a function of bounded variation on $[0,1]$.

The operators $D_{n}^{(\alpha)}(f ; x)$ also admit the integral representation

$$
D_{n}^{(\alpha)}(f ; x)=\int_{0}^{1} N_{n}^{(\alpha)}(x, t) f(t) d t, \quad x \in[0,1],
$$

where the kernel $N_{n}^{(\alpha)}(x, t)$ is given by

$$
N_{n}^{(\alpha)}(x, t)=(n+1) \sum_{k=0}^{n} p_{n, k}^{(\alpha)}(x) p_{n, k}(t) .
$$

Lemma 6. For a fixed $x \in(0,1)$ and sufficiently large $n$, we have

(i) $\vartheta_{n}(x, y)=\int_{0}^{y} N_{n}^{(\alpha)}(x, t) d t \leq \frac{2}{(n+2)} \frac{\gamma_{n}^{2}(x)}{(x-y)^{2}}, 0 \leq y<x$,

(ii) $1-\vartheta_{n}(x, z)=\int_{z}^{1} N_{n}^{(\alpha)}(x, t) d t \leq \frac{2}{(n+2)} \frac{\gamma_{n}^{2}(x)}{(z-x)^{2}}, x<z<1$.

Proof. (i) Using Lemma 5 we get

$$
\begin{aligned}
\vartheta_{n}(x, y) & =\int_{0}^{y} N_{n}^{(\alpha)}(x, t) d t \leq \int_{0}^{y}\left(\frac{x-t}{x-y}\right)^{2} N_{n}^{(\alpha)}(x, t) d t \\
& =D_{n}^{(\alpha)}\left((t-x)^{2} ; x\right)(x-y)^{-2} \leq \frac{2}{(n+2)} \frac{\gamma_{n}^{2}(x)}{(x-y)^{2}} .
\end{aligned}
$$

The proof of (ii) is similar hence the details are omitted. 
Theorem 7. Let $f \in D B V[0,1]$. Then for every $x \in(0,1)$ and sufficiently large $n$, we have

$$
\begin{aligned}
\left|D_{n}^{(\alpha)}(f ; x)-f(x)\right| \leq & \left|\frac{(1-2 x)}{n+2}\right| \frac{\left|f^{\prime}(x+)+f^{\prime}(x-)\right|}{2} \\
& +\sqrt{\frac{2}{(n+2)}} \gamma_{n}(x) \frac{\left|f^{\prime}(x+)-f^{\prime}(x-)\right|}{2} \\
& +\frac{2 \gamma_{n}^{2}(x)}{(n+2)} x^{-1} \sum_{k=1}^{[\sqrt{n}]} \bigvee_{x-(x / k)}^{x}\left(f_{x}^{\prime}\right)+\frac{x}{\sqrt{n}} \bigvee_{x-(x / \sqrt{n})}^{x}\left(f_{x}^{\prime}\right) \\
& +\frac{2 \gamma_{n}^{2}(x)}{(1-x)(n+2)} \sum_{k=1}^{[\sqrt{n}] x+((1-x) / k)} \bigvee_{x}\left(f_{x}^{\prime}\right) \\
& +\frac{(1-x)}{\sqrt{n}} \bigvee_{x}^{x+((1-x) / \sqrt{n})}\left(f_{x}^{\prime}\right),
\end{aligned}
$$

where $\bigvee_{a}^{b}\left(f_{x}^{\prime}\right)$ denotes the total variation of $f_{x}^{\prime}$ on $[a, b]$ and $f_{x}^{\prime}$ is defined by

$$
f_{x}^{\prime}(t)=\left\{\begin{array}{cc}
f^{\prime}(t)-f^{\prime}(x-), & 0 \leq t<x \\
0, & t=x \\
f^{\prime}(t)-f^{\prime}(x+) & x<t<1 .
\end{array}\right.
$$

Proof. Since $D_{n}^{(\alpha)}\left(e_{0} ; x\right)=1$, by applying (7.1), for every $x \in(0,1)$ we obtain

$$
\begin{aligned}
D_{n}^{(\alpha)}(f ; x)-f(x) & =\int_{0}^{1} N_{n}^{(\alpha)}(x, t)(f(t)-f(x)) d t \\
& =\int_{0}^{1} N_{n}^{(\alpha)}(x, t) \int_{x}^{t} f^{\prime}(u) d u d t .
\end{aligned}
$$

For any $f \in D B V[0,1]$, by (7.2) we may write

$$
\begin{aligned}
f^{\prime}(u)= & f_{x}^{\prime}(u)+\frac{1}{2}\left(f^{\prime}(x+)+f^{\prime}(x-)\right)+\frac{1}{2}\left(f^{\prime}(x+)-f^{\prime}(x-)\right) \operatorname{sgn}(u-x) \\
& +\delta_{x}(u)\left[f^{\prime}(u)-\frac{1}{2}\left(f^{\prime}(x+)+f^{\prime}(x-)\right)\right]
\end{aligned}
$$

where

$$
\delta_{x}(u)=\left\{\begin{array}{l}
1, u=x \\
0, u \neq x
\end{array}\right.
$$

Obviously,

$$
\int_{0}^{1}\left(\int_{x}^{t}\left(f^{\prime}(u)-\frac{1}{2}\left(f^{\prime}(x+)+f^{\prime}(x-)\right)\right) \delta_{x}(u) d u\right) N_{n}^{(\alpha)}(x, t) d t=0 .
$$


By (7.1) and simple calculations we have

$$
\begin{aligned}
\int_{0}^{1}\left(\int_{x}^{t} \frac{1}{2}\left(f^{\prime}(x+)+f^{\prime}(x-)\right)\right. & d u) N_{n}^{(\alpha)}(x, t) d t \\
& =\frac{1}{2}\left(f^{\prime}(x+)+f^{\prime}(x-)\right) \int_{0}^{1}(t-x) N_{n}^{(\alpha)}(x, t) d t \\
& =\frac{1}{2}\left(f^{\prime}(x+)+f^{\prime}(x-)\right) D_{n}^{(\alpha)}((t-x) ; x)
\end{aligned}
$$

and

$$
\begin{aligned}
& \mid \int_{0}^{1} N_{n}^{(\alpha)}(x, t) \\
& \left(\int_{x}^{t} \frac{1}{2}\left(f^{\prime}(x+)-f^{\prime}(x-)\right) \operatorname{sgn}(u-x) d u\right) d t \mid \\
& \leq \frac{1}{2}\left|f^{\prime}(x+)-f^{\prime}(x-)\right| \int_{0}^{1}|t-x| N_{n}^{(\alpha)}(x, t) d t \\
& \leq \frac{1}{2}\left|f^{\prime}(x+)-f^{\prime}(x-)\right| D_{n}^{(\alpha)}(|t-x| ; x) \\
& \leq \frac{1}{2}\left|f^{\prime}(x+)-f^{\prime}(x-)\right|\left(D_{n}^{(\alpha)}\left((t-x)^{2} ; x\right)\right)^{1 / 2}
\end{aligned}
$$

Considering Lemmas 3 and 5 and using (7.3), (7.4) we obtain the following estimate

$$
\begin{aligned}
\left|D_{n}^{(\alpha)}(f ; x)-f(x)\right| \leq & \frac{1}{2}\left|f^{\prime}(x+)+f^{\prime}(x-)\right|\left|\frac{1-2 x}{(n+2)}\right| \\
& +\frac{1}{2}\left|f^{\prime}(x+)-f^{\prime}(x-)\right| \sqrt{\frac{2}{(n+2)}} \gamma_{n}(x) \\
& +\mid \int_{0}^{x}\left(\int_{x}^{t} f_{x}^{\prime}(u) d u\right) N_{n}^{(\alpha)}(x, t) d t \\
& +\int_{x}^{1}\left(\int_{x}^{t} f_{x}^{\prime}(u) d u\right) N_{n}^{(\alpha)}(x, t) d t \mid
\end{aligned}
$$

Let

$$
\begin{aligned}
& F_{n}^{(\alpha)}\left(f_{x}^{\prime}, x\right)=\int_{0}^{x}\left(\int_{x}^{t} f_{x}^{\prime}(u) d u\right) N_{n}^{(\alpha)}(x, t) d t, \\
& G_{n}^{(\alpha)}\left(f_{x}^{\prime}, x\right)=\int_{x}^{1}\left(\int_{x}^{t} f_{x}^{\prime}(u) d u\right) N_{n}^{(\alpha)}(x, t) d t .
\end{aligned}
$$

To complete the proof, it is sufficient to estimate the terms $F_{n}^{(\alpha)}\left(f_{x}^{\prime}, x\right)$ and $G_{n}^{(\alpha)}\left(f_{x}^{\prime}, x\right)$. Since $\int_{a}^{b} d_{t} \vartheta_{n}(x, t) \leq 1$ for all $[a, b] \subseteq[0,1]$, using integration by parts 
and applying Lemma 6 with $y=x-(x / \sqrt{n})$, we have

$$
\begin{aligned}
\left|F_{n}^{(\alpha)}\left(f_{x}^{\prime}, x\right)\right| & =\left|\int_{0}^{x}\left(\int_{x}^{t} f_{x}^{\prime}(u) d u\right) d_{t} \vartheta_{n}(x, t)\right|=\left|\int_{0}^{x} \vartheta_{n}(x, t) f_{x}^{\prime}(t) d t\right| \\
& \leq\left(\int_{0}^{y}+\int_{y}^{x}\right)\left|f_{x}^{\prime}(t)\right|\left|\vartheta_{n}(x, t)\right| d t \\
& \leq \frac{2 \gamma_{n}^{2}(x)}{(n+2)} \int_{0}^{y} \bigvee_{t}^{x}\left(f_{x}^{\prime}\right)(x-t)^{-2} d t+\int_{y}^{x} \bigvee_{t}^{x}\left(f_{x}^{\prime}\right) d t \\
& \leq \frac{2 \gamma_{n}^{2}(x)}{(n+2)} \int_{0}^{y} \bigvee_{t}^{x}\left(f_{x}^{\prime}\right)(x-t)^{-2} d t+\frac{x}{\sqrt{n}} \bigvee_{x-(x / \sqrt{n})}^{x}\left(f_{x}^{\prime}\right) .
\end{aligned}
$$

By the substitution of $u=x /(x-t)$, we obtain

$$
\begin{aligned}
\frac{2 \gamma_{n}^{2}(x)}{(n+2)} \int_{0}^{x-(x / \sqrt{n})}(x-t)^{-2} \bigvee_{t}^{x}\left(f_{x}^{\prime}\right) d t & =\frac{2 \gamma_{n}^{2}(x)}{(n+2)} x^{-1} \int_{1}^{\sqrt{n}} \bigvee_{x-(x / u)}^{x}\left(f_{x}^{\prime}\right) d u \\
& \leq \frac{2 \gamma_{n}^{2}(x)}{(n+2)} x^{-1} \sum_{k=1}^{[\sqrt{n}]} \int_{k}^{k+1} \bigvee_{x-(x / k)}^{x}\left(f_{x}^{\prime}\right) d u \\
& \leq \frac{2 \gamma_{n}^{2}(x)}{(n+2)} x^{-1} \sum_{k=1}^{[\sqrt{n}]} \bigvee_{x-(x / k)}^{x}\left(f_{x}^{\prime}\right)
\end{aligned}
$$

Thus,

$$
\left|F_{n}^{(\alpha)}\left(f_{x}^{\prime}, x\right)\right| \leq \frac{2 \gamma_{n}^{2}(x)}{(n+2)} x^{-1} \sum_{k=1}^{[\sqrt{n}]} \bigvee_{x-(x / k)}^{x}\left(f_{x}^{\prime}\right)+\frac{x}{\sqrt{n}} \bigvee_{x-(x / \sqrt{n})}^{x}\left(f_{x}^{\prime}\right)
$$

Using integration by parts and applying Lemma 6 with $z=x+((1-x) / \sqrt{n})$, we have

$$
\begin{aligned}
\left|G_{n}^{(\alpha)}\left(f_{x}^{\prime}, x\right)\right|= & \left|\int_{x}^{1}\left(\int_{x}^{t} f_{x}^{\prime}(u) d u\right) N_{n}^{(\alpha)}(x, t) d t\right| \\
= & \mid \int_{x}^{z}\left(\int_{x}^{t} f_{x}^{\prime}(u) d u\right) d_{t}\left(1-\vartheta_{n}(x, t)\right) \\
& +\int_{z}^{1}\left(\int_{x}^{t} f_{x}^{\prime}(u) d u\right) d_{t}\left(1-\vartheta_{n}(x, t)\right) \mid \\
= & \mid\left[\int_{x}^{t} f_{x}^{\prime}(u)\left(1-\vartheta_{n}(x, t)\right) d u\right]_{x}^{z}-\int_{x}^{z} f_{x}^{\prime}(t)\left(1-\vartheta_{n}(x, t)\right) d t
\end{aligned}
$$




$$
\begin{aligned}
& +\int_{z}^{1}\left(\int_{x}^{t} f_{x}^{\prime}(u) d u\right) d_{t}\left(1-\vartheta_{n}(x, t)\right) \mid \\
= & \mid \int_{x}^{z} f_{x}^{\prime}(u) d u\left(1-\vartheta_{n}(x, z)\right)-\int_{x}^{z} f_{x}^{\prime}(t)\left(1-\vartheta_{n}(x, t)\right) d t \\
& +\left[\int_{x}^{t} f_{x}^{\prime}(u) d u\left(1-\vartheta_{n}(x, t)\right)\right]_{z}^{1}-\int_{z}^{1} f_{x}^{\prime}(t)\left(1-\vartheta_{n}(x, t)\right) d t \mid \\
= & \left|\int_{x}^{z} f_{x}^{\prime}(t)\left(1-\vartheta_{n}(x, t)\right) d t+\int_{z}^{1} f_{x}^{\prime}(t)\left(1-\vartheta_{n}(x, t)\right) d t\right| \\
\leq & \frac{2 \gamma_{n}^{2}(x)}{(n+2)} \int_{z}^{1} \bigvee_{x}^{t}\left(f_{x}^{\prime}\right)(t-x)^{-2} d t+\int_{x}^{z} \bigvee_{x}^{t}\left(f_{x}^{\prime}\right) d t \\
= & \frac{2 \gamma_{n}^{2}(x)}{(n+2)} \int_{x+((1-x) / \sqrt{n})}^{1} \bigvee_{x}^{t}\left(f_{x}^{\prime}\right)(t-x)^{-2} d t \\
& +\frac{(1-x)}{\sqrt{n}} \bigvee_{x}^{x+((1-x) / \sqrt{n})}\left(f_{x}^{\prime}\right) .
\end{aligned}
$$

By the substitution of $v=(1-x) /(t-x)$, we get

$$
\begin{aligned}
\left|G_{n}^{(\alpha)}\left(f_{x}^{\prime}, x\right)\right| \leq & \frac{2 \gamma_{n}^{2}(x)}{(n+2)} \int_{1}^{\sqrt{n} x+((1-x) / v)} \bigvee_{x}^{\prime}\left(f_{x}^{\prime}\right)(1-x)^{-1} d v \\
& +\frac{(1-x)}{\sqrt{n}} \bigvee_{x}^{x+((1-x) / \sqrt{n})}\left(f_{x}^{\prime}\right) \\
\leq & \frac{2 \gamma_{n}^{2}(x)}{(1-x)(n+2)} \sum_{k=1}^{[\sqrt{n}]} \int_{k}^{k+1} \bigvee_{x}^{x+((1-x) / v)}\left(f_{x}^{\prime}\right) d v \\
& +\frac{(1-x)}{\sqrt{n}} \bigvee_{x}^{x+((1-x) / \sqrt{n})}\left(f_{x}^{\prime}\right) \\
= & \frac{2 \gamma_{n}^{2}(x)}{(1-x)(n+2)} \sum_{k=1}^{[\sqrt{n}] x+((1-x) / k)} \bigvee_{x}^{\left(f_{x}^{\prime}\right)} \\
& +\frac{(1-x)}{\sqrt{n}} \bigvee_{x}^{x+((1-x)) / \sqrt{n}}\left(f_{x}^{\prime}\right) .
\end{aligned}
$$

Combining the estimates (7.5)-(7.7), we get the required result. 


\section{REFERENCES}

[1] U. Abel, V. Gupta, and M. Ivan, "Asymptotic approximation of functions and their derivatives by generalized Baskakov-Szász-Durrmeyer operators," Anal. Theory Appl., vol. 21, no. 1, pp. 15-26, 2005, doi: 10.1007/BF02835246. [Online]. Available: http://dx.doi.org/10.1007/BF02835246

[2] T. Acar, "Asymptotic formulas for generalized Szász-Mirakyan operators," Appl. Math. Comput., vol. 263, pp. 233-239, 2015, doi: 10.1016/j.amc.2015.04.060. [Online]. Available: http://dx.doi.org/10.1016/j.amc.2015.04.060

[3] T. Acar and A. Aral, "On pointwise convergence of q-Bernstein operators and their q-derivatives," Numer. Funct. Anal. Optim., vol. 36, no. 3, pp. 287-304, 2015, doi: 10.1080/01630563.2014.970646. [Online]. Available: http://dx.doi.org/10.1080/01630563.2014. 970646

[4] T. Acar, A. Aral, and I. Raşa, "Modified Bernstein-Durrmeyer operators," General Mathematics, vol. 22, pp. 27-41, 2014.

[5] T. Acar, V. Gupta, and A. Aral, "Rate of convergence for generalized Szász operators," Bull. Math. Sci., vol. 1, no. 1, pp. 99-113, 2011, doi: 10.1007/s13373-011-0005-4. [Online]. Available: http://dx.doi.org/10.1007/s13373-011-0005-4

[6] T. Acar and G. Ulusoy, “Approximation by modified Szász-Durrmeyer operators," Period. Math. Hungar., vol. 72, no. 1, pp. 64-75, 2016, doi: 10.1007/s10998-015-0091-2. [Online]. Available: http://dx.doi.org/10.1007/s10998-015-0091-2

[7] P. N. Agrawal, M. Goyal, and A. Kajla, "q-Berntsein-Schurer-Kantorovich type operators," Boll. Unione Mat. Ital., vol. 8, pp. 169-180, 2015.

[8] P. N. Agrawal, V. Gupta, A. Sathish Kumar, and A. Kajla, "Generalized Baskakov-Szász type operators," Appl. Math. Comput., vol. 236, pp. 311-324, 2014, doi: 10.1016/j.amc.2014.03.084. [Online]. Available: http://dx.doi.org/10.1016/j.amc.2014.03.084

[9] D. Cárdenas-Morales, P. Garrancho, and I. Raşa, "Asymptotic formulae via a Korovkin-type result," Abstr. Appl. Anal., pp. Art. ID 217464, 12, 2012, doi: 10.1155/2012/217464. [Online]. Available: http://dx.doi.org/10.1155/2012/217464

[10] X. Chen, J. Tan, Z. Liu, and J. Xie, "Approximation of functions by a new family of generalized Bernstein operators," J. Math. Anal. Appl., vol. 450, no. 1, pp. 244-261, 2017, doi: 10.1215/20088752-3764507. [Online]. Available: http://dx.doi.org/10.1016/j.jmaa.2016.12.075

[11] R. A. DeVore and G. G. Lorentz, Constructive Approximation, ser. Grundlehren der Mathematischen Wissenschaften [Fundamental Principles of Mathematical Sciences]. SpringerVerlag, Berlin, 1993, vol. 303. [Online]. Available: http://dx.doi.org/10.1007/978-3-662-02888-9. doi: 10.1007/978-3-662-02888-9

[12] Z. Ditzian and V. Totik, Moduli of smoothness, ser. Springer Series in Computational Mathematics. Springer-Verlag, New York, 1987, vol. 9. [Online]. Available: http: //dx.doi.org/10.1007/978-1-4612-4778-4. doi: 10.1007/978-1-4612-4778-4

[13] A. D. Gadjiev and A. M. Ghorbanalizadeh, "Approximation properties of a new type Bernstein-Stancu polynomials of one and two variables," Appl. Math. Comput., vol. 216, no. 3, pp. 890-901, 2010, doi: 10.1016/j.amc.2010.01.099. [Online]. Available: http://dx.doi.org/10.1016/j.amc.2010.01.099

[14] M. Goyal, V. Gupta, and P. N. Agrawal, "Quantitative convergence results for a family of hybrid operators,” Appl. Math. Comput., vol. 271, pp. 893-904, 2015, doi: 10.1016/j.amc.2015.08.122. [Online]. Available: http://dx.doi.org/10.1016/j.amc.2015.08.122

[15] V. Gupta and A. M. Acu, "Direct results for certain summation-integral type Baskakov-Szász operators," Results. Math., pp. 10.1007/s00 025-016-0603-2., 2017. 
[16] V. Gupta, A. M. Acu, and D. F. Sofonea, "Approximation of Baskakov type Pólya-Durrmeyer operators," Appl. Math. Comput., vol. 294, pp. 318-331, 2017, doi: 10.1016/j.amc.2016.09.012. [Online]. Available: http://dx.doi.org/10.1016/j.amc.2016.09.012

[17] V. Gupta and R. P. Agarwal, Convergence Estimates in Approximation Theory. Springer, Cham, 2014. [Online]. Available: http://dx.doi.org/10.1007/978-3-319-02765-4. doi: 10.1007/978-3319-02765-4

[18] V. Gupta and T. M. Rassias, "Lupaş-Durrmeyer operators based on Polya distribution," Banach J. Math. Anal., vol. 8, no. 2, pp. 146-155, 2014. [Online]. Available: http: //projecteuclid.org/euclid.bjma/1396640060

[19] A. Kajla and T. Acar, "A new modification of Durrmeyer type mixed hybrid operators," Carpathian J. Math., vol. 34, no. 1, pp. 47-56, 2018.

[20] A. Kajla, A. M. Acu, and P. N. Agrawal, "Baskakov-Szász-type operators based on inverse Pólya-Eggenberger distribution,” Ann. Funct. Anal., vol. 8, no. 1, pp. 106-123, 2017, doi: 10.1215/20088752-3764507. [Online]. Available: http://dx.doi.org/10.1215/20088752-3764507

[21] A. Kajla and P. N. Agrawal, "Szász-Durrmeyer type operators based on Charlier polynomials," Appl. Math. Comput., vol. 268, pp. 1001-1014, 2015, doi: 10.1016/j.amc.2015.06.126. [Online]. Available: http://dx.doi.org/10.1016/j.amc.2015.06.126

[22] B. Lenze, "On Lipschitz-type maximal functions and their smoothness spaces," Nederl. Akad. Wetensch. Indag. Math., vol. 50, no. 1, pp. 53-63, 1988.

[23] M. Mursaleen, K. J. Ansari, and A. Khan, "On $(p, q)$-analogue of Bernstein operators," Appl. Math. Comput., vol. 266, pp. 874-882, 2015, doi: 10.1016/j.amc.2015.04.090. [Online]. Available: http://dx.doi.org/10.1016/j.amc.2015.04.090

[24] M. A. Özarslan and H. Aktu glu, "Local approximation properties for certain King type operators," Filomat, vol. 27, no. 1, pp. 173-181, 2013, doi: 10.2298/FIL1301173O. [Online]. Available: http://dx.doi.org/10.2298/FIL13011730

[25] M. Wang, D. Yu, and P. Zhou, "On the approximation by operators of Bernstein-Stancu types," Appl. Math. Comput., vol. 246, pp. 79-87, 2014, doi: 10.1016/j.amc.2014.08.015. [Online]. Available: http://dx.doi.org/10.1016/j.amc.2014.08.015

Authors' addresses

Arun Kajla

Department of Mathematics, Central University of Haryana, Haryana-123031, India

E-mail address: rachitkajla47@gmail.com

\section{Tuncer Acar}

Department of Mathematics,Faculty of Science, Selcuk University, Selcuklu, Konya, 42003, Turkey

E-mail address: tunceracar@ymail.com 